\title{
INTERPRETACIÓN DE LOS DAÑOS Y COLAPSOS EN EDIFICACIONES OBSERVADOS EN LA CIUDAD DE MÉXICO EN EL TERREMOTO DEL 19 DE SEPTIEMBRE 2017
}

\author{
Mario E. Rodríguez ${ }^{(1)}$ \\ RESUMEN
}

\begin{abstract}
En este trabajo se interpretan los daños y colapsos de edificaciones observados en el terremoto del 19 de septiembre 2017 en la Ciudad de México, los que ocurrieron en su mayoría en edificaciones a base de marcos que experimentaron el terremoto de 1985. Se encontró que las demandas espectrales para registros obtenidos en 1985 y 2017 son comparables, lo que sugiere explicar por qué llegaron a colapsos o daños severos en 2017 y no en 1985 . Se emplea un índice de daño por sismo, $I_{d}$, propuesto anteriormente por el autor, el cual toma en cuenta el daño por sismo acumulado. Se calculan valores de $I_{d}$ para edificaciones considerando registros de 1985 y registros de 2017, así como para el efecto combinado de estos registros. Los resultados indican que los colapsos y daños observados en marcos en 2017, se explican considerando el efecto de daño por sismo acumulado. Se muestra además la conveniencia del empleo de edificaciones con muros estructurales para reducir el potencial de daños o colapsos ante terremotos fuertes. Se dan recomendaciones de cambios en normativas de construcción en la Ciudad de México y en el país.
\end{abstract}

Palabras clave: Daños y colapsos en edificios; terremotos en México; daño acumulado

\section{INTERPRETATION OF DAMAGE AND COLLAPSES IN BUILDINGS OBSERVED IN MEXICO CITY IN THE SEPTEMBER 19 EARTHQUAKE}

\begin{abstract}
This study evaluates the damage and collapses of buildings in the September 2017 earthquake in Mexico City. These buildings were of the frame type, they were also subjected to the September 1985 Mexico and little or no damage was observed in that event. It was found that the spectral demands for typical records registered in 1985 and 2017 were not much different, which suggests the need to explain why these buildings collapsed or had severe damage in 2017 and not in 1985. This building behavior was analyzed using a seismic damage index previously proposed by the author, $I_{d}$, which considers the effect of cumulative damage. Index $I_{d}$ was computed for typical 1985 and 2017 ground motion records, as well as for a record resulting of the combination of both the 1985 record and the 2017 record. Results indicate that the observed damage and collapses of buildings in the September 2017 earthquake can be explained considering the effect of cumulative seismic damage. Furthermore, it was found that dual systems are much more effective for reducing damage potential of buildings than frame buildings. Recommendations are given for possible improvements of seismic building codes in Mexico.
\end{abstract}

Keywords: Damage and collapse in buildings; earthquakes in Mexico; cumulative damage

Artículo recibido el 24 de septiembre de 2018 y aprobado para su publicación el 7 de octubre de 2019. Se aceptarán comentarios y/o discusiones hasta cinco meses después de su publicación

(1) Profesor-Investigador, Instituto de Ingeniería, UNAM, Ciudad Universitaria, CP 04510,México DF, mrod@unam.mx 


\section{NOTAS GENERALES REFERENTES AL TERREMOTO OCURRIDO EL 19 DE SEPTIEMBRE DE 2017 Y DAÑOS OBSERVADOS}

El 19 de septiembre 2017 ocurrió un terremoto $\mathrm{M}_{\mathrm{w}} 7.1$ en México del tipo intraplaca, con epicentro en el Estado de Morelos, a alrededor de $120 \mathrm{~km}$ de la Ciudad de México. El terremoto causó el colapso de aproximadamente 50 edificaciones, número estimado de acuerdo con los datos obtenidos por ingenieros especialistas en estructuras distribuidos en alrededor de 30 brigadas que inspeccionaron la mayor parte de daños en edificaciones en la ciudad de México debidos a este terremoto. Esta labor la llevó a cabo sociedades técnicas como la Sociedad Mexicana de Ingeniería Estructural (SMIE) y fue coordinada por el Colegio de Ingenieros Civiles de México (CICM).

El número de colapsos en el terremoto de 2017 en su mayoría se observó en edificaciones con menos de 10 niveles. La estadística de daños y colapsos en el referido terremoto (Berrón, 2018) evidencia que estas edificaciones ya habían experimentado el terremoto $\mathrm{M}_{\mathrm{w}} 8.1$ del 19 de Septiembre de 1985, el cual fue del tipo de subducción interplaca. Estos colapsos produjeron la muerte de más de 200 personas en la Ciudad de México. También se observaron daños severos estructurales y no estructurales en edificaciones, las que en su mayoría son de menos de 10 niveles y fueron construidas antes de 1985. Entre otras características en la mayoría de estas edificaciones, se puede mencionar que el sistema estructural típico es a base de marcos de concreto reforzado, con o sin muros de mampostería divisorios, con la llamada "planta baja débil", causada por la presencia de estacionamientos a nivel del terreno, sin o con pocos muros de mampostería en este nivel. Estas edificaciones a base de marcos, por su naturaleza podrían tener insuficiente rigidez lateral. Además, se puede considerar que el detallado de refuerzo en ellas está por debajo de los estándares internacionales para el diseño de estructuras en zona sísmicas, esto principalmente porque desde antes de 1985 y después de esta fecha, la normativa por sismo en la ciudad de México permitía la construcción de marcos de concreto reforzado sin los requisitos especiales de diseño para elementos estructurales en zonas sísmicas, especificados en el Capítulo 21 de versiones anteriores al ACI 318-14, y ahora en esta última normativa en el Capítulo 18. Esta deficiencia en esa normativa se repite en las actuales normativas por sismo en la Ciudad de México (NTCS, 2017),

La Fig. 1 muestra la distribución de colapsos de edificios observados en el terremoto del 19 de Septiembre 2017, los que se ubican en el mapa de igual periodos del suelo en la Ciudad de México. Estos datos de colapsos han sido obtenidos de la información de brigadas organizadas por el CICM.

Estas curvas de igual periodos del suelo mostradas en la Fig. 1 fueron propuestas por Martínez-González et al. (2015) y es el resultado de mediciones de las características de los suelos de la Ciudad de México llevadas a cabo en los últimos cinco años. Esta información ha sido propuesta para la zonificación sísmica de las NTCS 2017 (Martínez-Gonzales et al., 2015).

La Fig. 1 muestra una zona de suelo de la Ciudad de México con color blanco, limitada en el lado Oeste por la curva de igual periodo dominante del suelo con valor 1s, y en el lado Este esta zona está limitada por la curva de igual periodo con valor 1.5s. De acuerdo con las NTCS (2004), la zona de suelo compresible en la Ciudad de México empieza en la curva de igual periodo de 1s. Como muestra la fig. 1, la zona de colapsos de edificaciones en el terremoto del 19 de septiembre de 2017 se ubica principalmente entre la curva de igual periodo de $1 \mathrm{~s}$ y la de $1.5 \mathrm{~s}$, la que corresponde aproximadamente a la zona IIIa de la zonificación sísmica de la Ciudad de México especificada por las normativas por sismo aprobadas en 2004 (NTCS, 2004). 


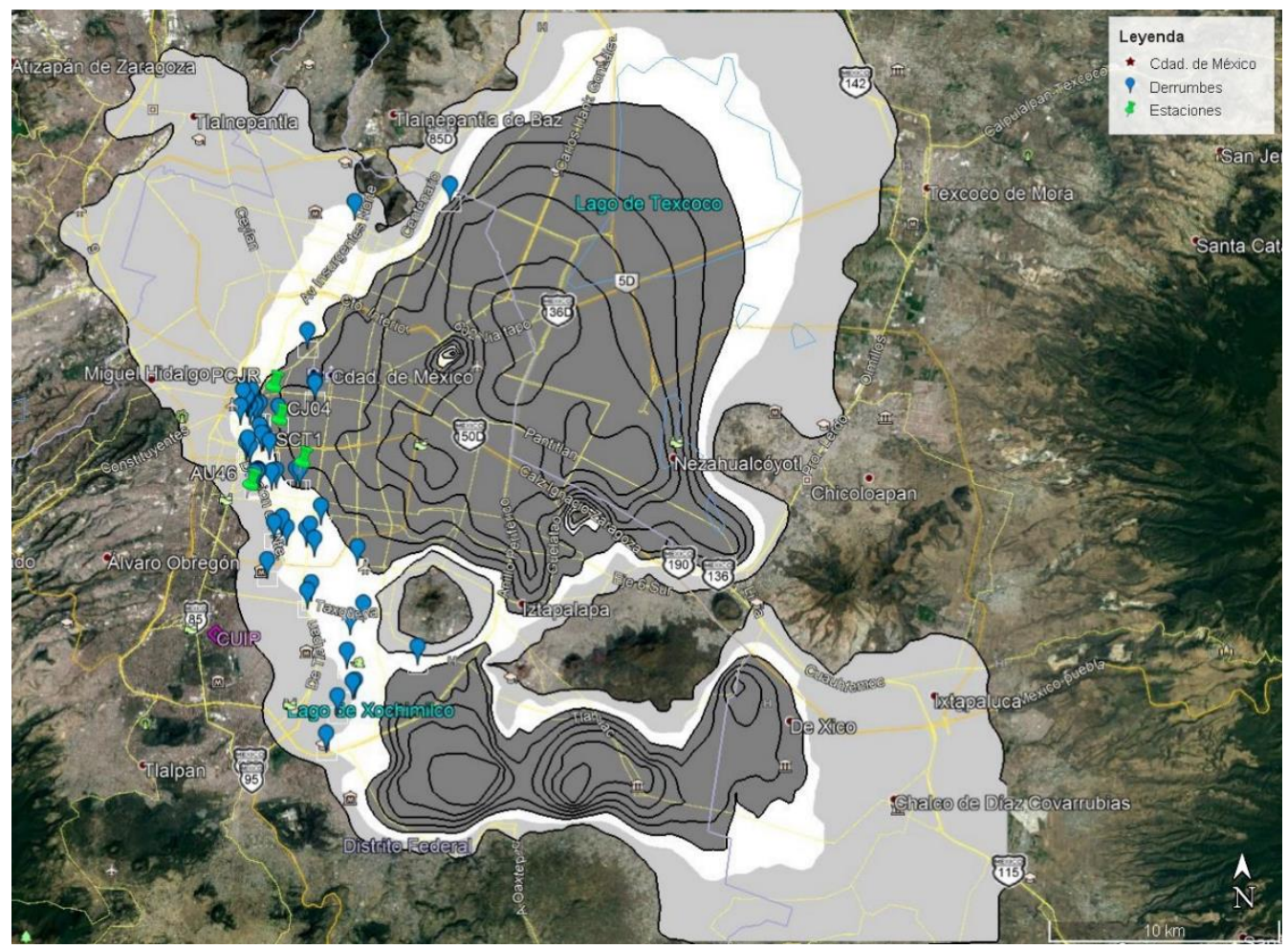

Figura 1. Ubicación de colapsos observados en la Ciudad de México en el terremoto del 19 de Septiembre de 2017 (en azul), ubicación de estaciones cercanas a estos colapsos (en verde), estación CU (CUIP) en Ciudad Universitaria, y mapa de curvas de igual periodos del suelo

\section{OBJETIVOS DE ESTE TRABAJO}

En este trabajo se intenta contribuir a la discusión de las razones de los referidos daños o colapsos, con el fin de llegar a conclusiones que definan procedimientos específicos para la rehabilitación de los edificios afectados por este terremoto, así como para definir posibles cambios necesarios en normativas de construcción en la Ciudad de México y en el país.

\section{CARACTERÍSTICAS DE ESPECTROS DE RESPUESTA PARA LOS REGISTROS DE ACELERACIONES OBTENIDOS EN LAS ESTACIONES CU Y SCT, EN LOS TERREMOTOS DEL 19 DE SEPTIEMBRE DE 1985 Y 19 DE SEPTIEMBRE DE 2017}

\section{Estación CU}

La estación CU, identificada como CUIP en la Fig. 1, se ubica en la zona de suelo firme de la ciudad de México. En esta estación, así como en la estación SCT, que se ubica en suelo blando y que se comenta más adelante, se obtuvieron registros de aceleraciones tanto para el terremoto de 1985 como para el de 2017. El análisis de la respuesta espectral para estos registros es un primer paso para entender el comportamiento observado en edificaciones en el último terremoto.

La Fig. 2a permite una comparativa de espectros elásticos de pseudo-aceleraciones, calculados con un factor de porcentaje de amortiguamiento crítico, $\xi$, igual a $5 \%$, para los registros de $\mathrm{CU}$ obtenidos en los terremotos 
de 1985 y 2017. En estos resultados se aprecia que los valores de $S_{a}$ para el registro en CU en 2017 son bastante mayores que los valores de $S_{a}$ en 1985 en la zona de periodos menores que $0.5 \mathrm{~s}$. Esta diferencia se debe a que el epicentro del terremoto de 2017 ocurrió en una distancia a la Ciudad de México menor que la observada en el terremoto de 1985, así como a las características de los terremotos de intraplaca, como fue el del evento de 2017, que se comenta en la sección siguiente. La Fig. 2b muestra espectros de desplazamientos para respuesta lineal, $S_{d}$, para los registros de 1985 y 2017 en CU. La diferencia entre estas respuestas espectrales de desplazamiento para los registros en $\mathrm{CU}$ en general es menos marcada que para el caso de pseudo-aceleraciones mostrada en la Fig. 2a.

$$
S_{a}, \mathrm{CU} 1985 \text { y } 2017, \xi=5 \%
$$

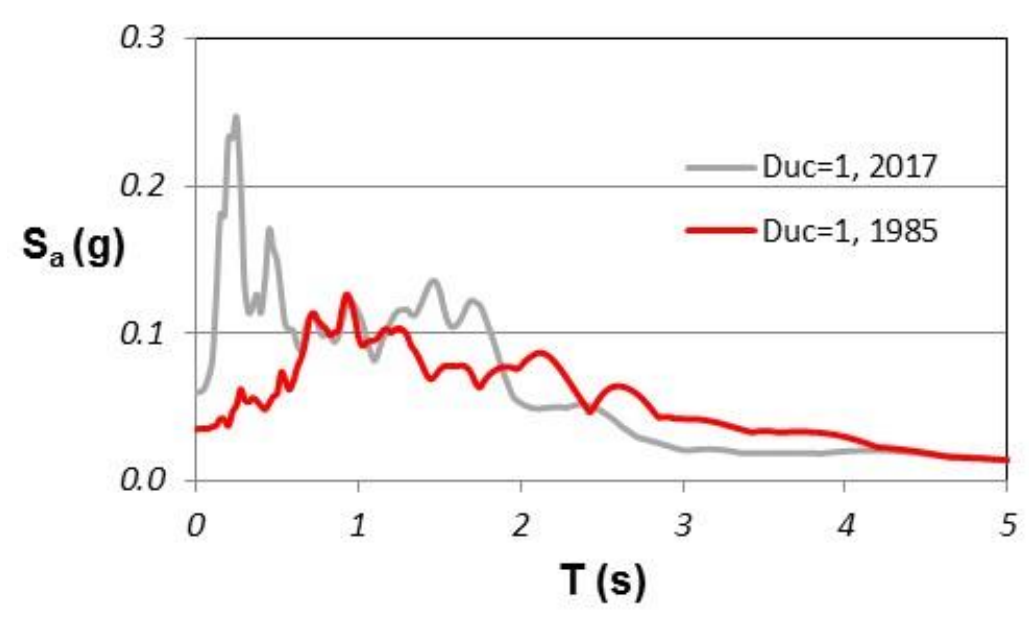

(a) Espectros $S_{a}$

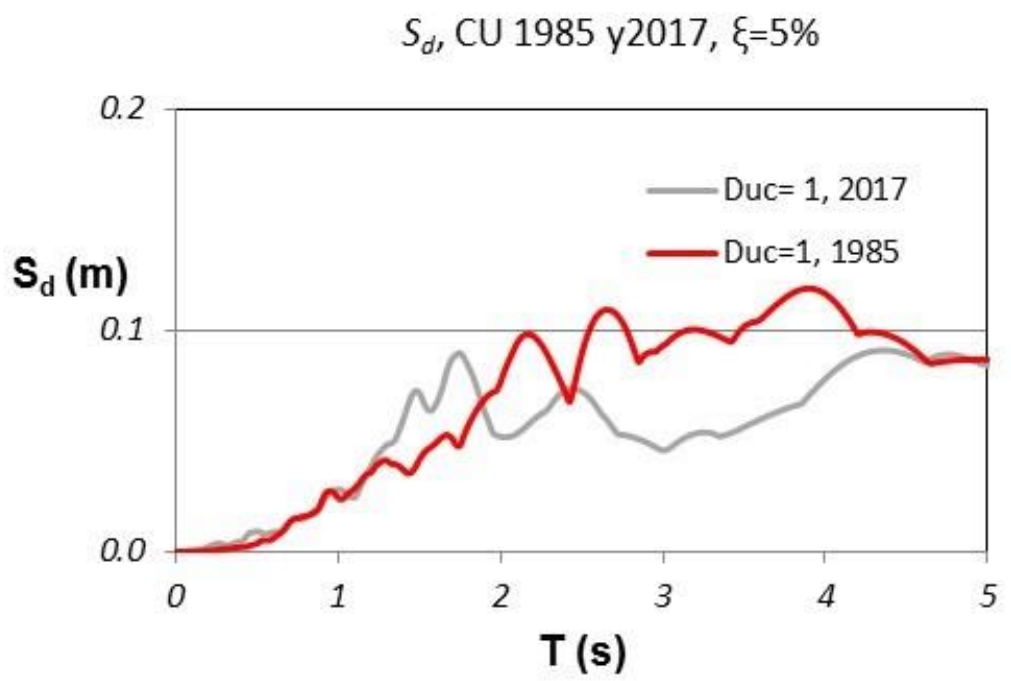

(b) Espectros $S_{d}$

Figura 2. Ordenadas elásticas $S_{a}$ y $S_{d}$ obtenidas con registros en la estación CU en los terremotos de 1985 y 2017 
Esta característica de la respuesta espectral que se muestra en la Fig. 2 para la estación CU, de mayores aceleraciones espectrales en la zona de periodos cortos, para el terremoto intraplaca, respecto a las del tipo interplaca de 1985, ha sido también observada en respuestas espectrales para movimientos debidos al terremoto tipo intraplaca del 11 de Enero de 1997, en la costa del Pacífico, en el estado de Michoacán (Rodriguez et al., 1999). Son de interés los registros de aceleraciones obtenidos en la estación Caleta de Campos en este terremoto. En un poblado cercano a esta estación se observaron daños en viviendas de mampostería en el terremoto de 1997 y no en el de 1985 (Rodriguez et al., 1999). Es de utilidad analizar las respuestas espectrales correspondientes al terremoto intraplaca de 1999 con las obtenidas con el registro de aceleraciones en esta misma estación en el terremoto interplaca del 19 de Septiembre de 1985. Los resultados para $S_{a}$ en el comportamiento elástico que permiten llevar a cabo esta comparativa se muestran en la Fig. 3 (Rodriguez et al., 1999). En ella se aprecia que, de manera análoga al caso de los registros en CU en 1985 y 2017 , en la zona de periodos menores que $0.5 \mathrm{~s}$, las ordenadas espectrales, $S_{a}$, para el registro de 1997 del terremoto intraplaca son bastante mayores que las correspondientes al terremoto interplaca de 1985.

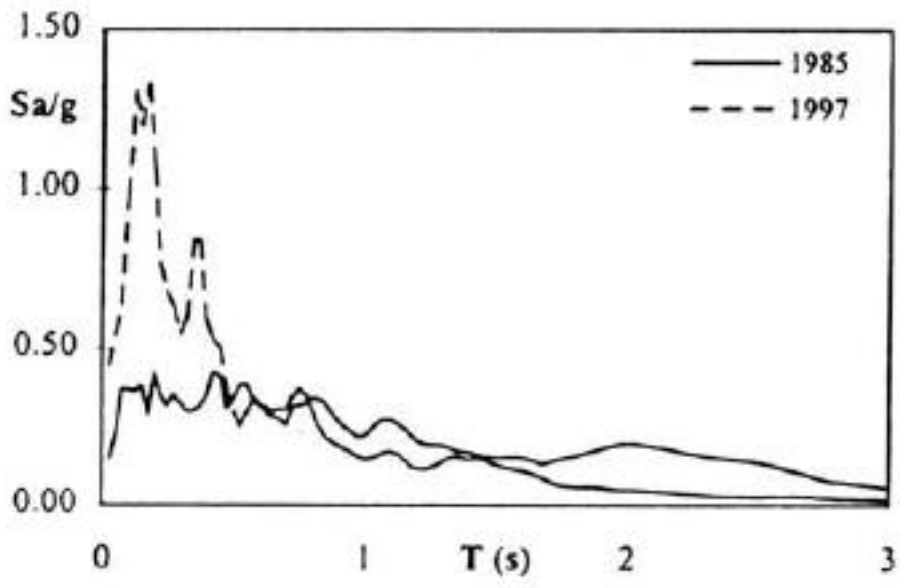

Figura 3. Ordenadas $S_{a}$ correspondientes a los registros de aceleraciones en la estación Caleta de Campos en los terremotos del 19 de septiembre de 1985 y del 11 de enero 1997 (Rodríguez et al., 1999)

La Fig. 4 muestra nuevamente los espectros de respuesta lineal de aceleraciones $S_{a}$ y de desplazamientos $S_{d}$ para el registro de 2017 en CU, obtenidos con $\xi=5 \%$, los que se comparan con las ordenadas espectrales de respuesta lineal especificadas para el tipo de suelo en CU por la Norma Técnica Complementaria por Sismo de 2004 (NTCS, 2004), vigente en la Ciudad de México en la fecha del terremoto de 2017. Los espectros de diseño de esta normativa no son del tipo transparente (están reducidos por el llamado factor de sobrerresistencia), por lo que las ordenadas en la Fig. 4 se han obtenido multiplicando las ordenadas espectrales especificadas por las NTCS de 2004 (NTCS, 2004) por un valor estimado del factor de probable sobrerresistencia, $\Omega$, para el cual en este análisis se ha considerado el valor de 2 . Los resultados de la Fig. 4 indican que las ordenadas espectrales para el registro en CU de 2017 no excedieron las ordenadas espectrales de diseño especificadas por las NTCS de 2004 (NTCS, 2004). Esto es congruente con la ausencia de colapsos o daños importantes en estructuras observado en zonas de suelo firme con características comparables a las del suelo de la estación en CU. 
$S_{a}$, CU 2017 y NTCS 2004, $\xi=5 \%$

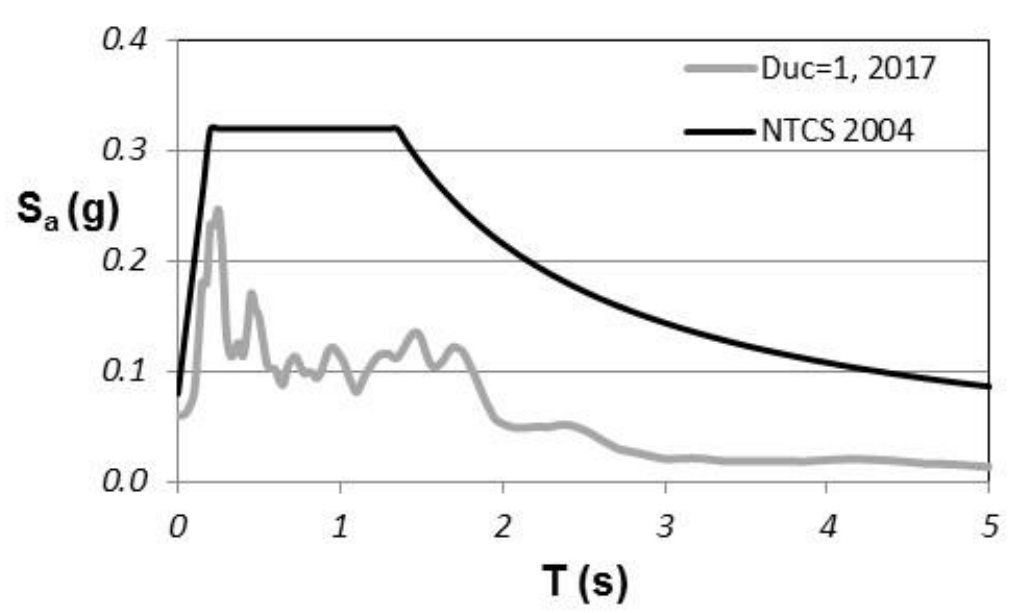

(a) Espectros $S_{a}$

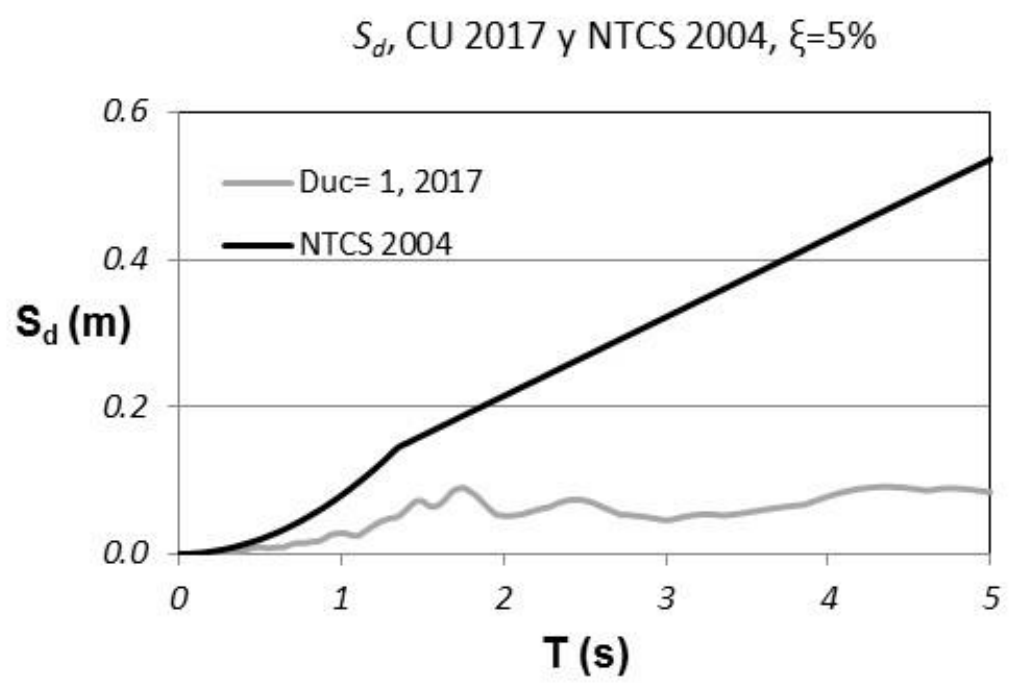

(b) Espectros $S_{d}$

Figura 4. Ordenadas espectrales de respuesta lineal $S_{a}$ y $S_{d}$ para los registros de la estación CU en el terremoto de 2017, y ordenadas especificadas por la NTCS 2004 para suelo firme

\section{Estación SCT}

Esta estación se ubica en el edificio de la Secretaría de Comunicaciones y Transporte (SCT) en la zona clasificada como suelo blando, Zona III, de acuerdo con las NTCS (2004). Este edificio se encuentra a menos de $1 \mathrm{~km}$ de la ubicación de algunos edificios con derrumbes debidos a este terremoto, ver Fig. 1. La Fig. 1, además de la estación SCT, muestra la ubicación de tres estaciones cercanas a la zona de daños que registraron aceleraciones en el terremoto de 2017, pero que no existían en el terremoto de 1985. En la zona de daños o cercana a ésta, sólo en la estación SCT se han registrados aceleraciones en ambos terremotos, 
de 1985 y 2017, por lo que su análisis es de importancia para tratar de comprender mejor los motivos de colapsos o daños severos observados en el terremoto de 2017. De acuerdo con este mapa, a esta estación le correspondería un periodo dominante del terreno igual a aproximadamente $1.8 \mathrm{~s}$, valor menor que el valor de 2 s que tenía en 1985, lo cual es el resultado del asentamiento regional del suelo en el tiempo en la Ciudad de México (Martínez-Gonzales et al., 2015). La Fig. 5 muestra ordenadas espectrales de repuesta lineal para $S_{a}$ y $S_{d}$ obtenidas para el registro SCT de 2017, las cuales se comparan con las que resultan de emplear las ordenadas especificadas por las NTCS (2004) para la zona de suelo blando que corresponde a la ubicación de la estación SCT. Por ser estos espectros de diseño del tipo no transparente, sus ordenadas se han multiplicado por el factor de sobrerresistencia $\Omega=2$. Los resultados de la Fig 5 muestran que como en el caso del registro de $\mathrm{CU}$, en suelo firme, las demandas espectrales para la respuesta lineal del registro SCT 2017 son menores que las demandas de diseño de la normativa vigente en la Ciudad de México en la fecha del terremoto de 2017.

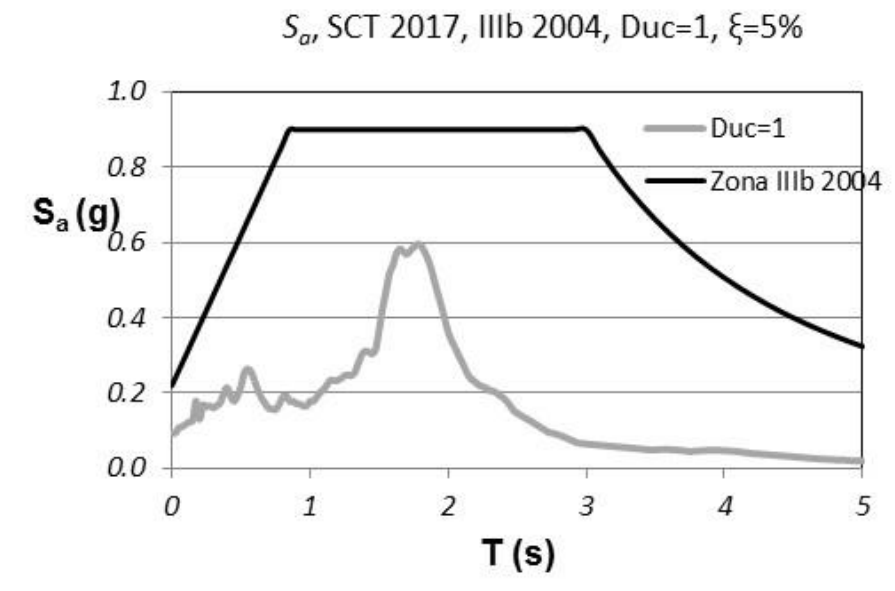

(a) Espectros $S_{a}$

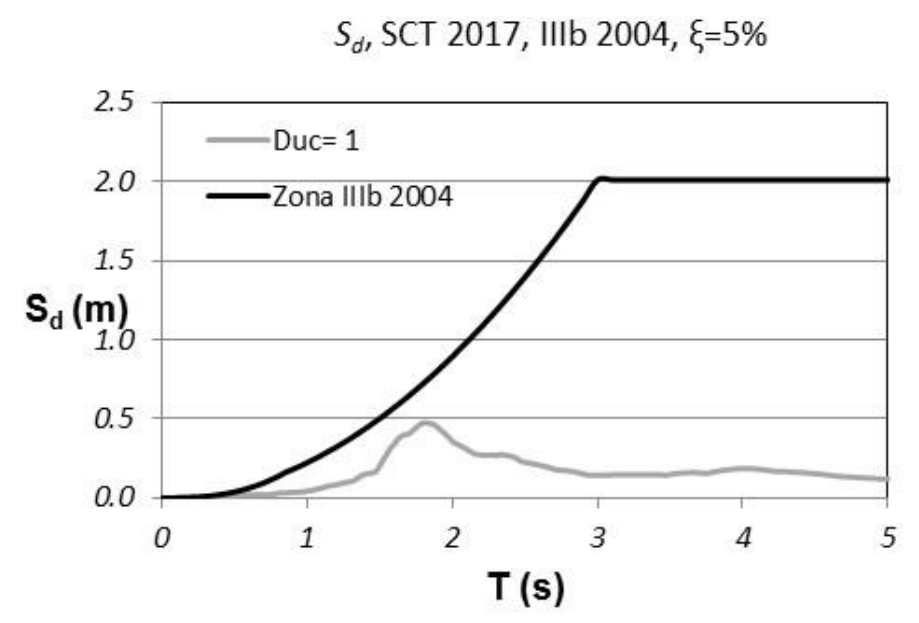

(b) Espectros $S_{d}$

Figura 5. Ordenadas elásticas $S_{a}$ y $S_{d}$ obtenidas con los registros de la estación SCT en el terremoto de 2017, y ordenadas especificadas por la NTCS 2004 para suelo blando IIIb 
En lo que sigue se comparan las ordenadas de aceleraciones espectrales $S_{a}$ para los registros de aceleraciones obtenidos en la estación SCT en los terremotos de 1985 y 2017. La Fig. 6 muestra las ordenadas de aceleraciones espectrales $S_{a}$ para cada uno de estos registros, para la respuesta lineal, $\mu=1$, así como para el caso de respuesta inelástica, con una ductilidad de desplazamiento $\mu=2$. Se aprecia que en la zona de periodos menores que alrededor de $1.5 \mathrm{~s}$, las ordenadas espectrales correspondientes a los registros de ambos terremotos son comparables.

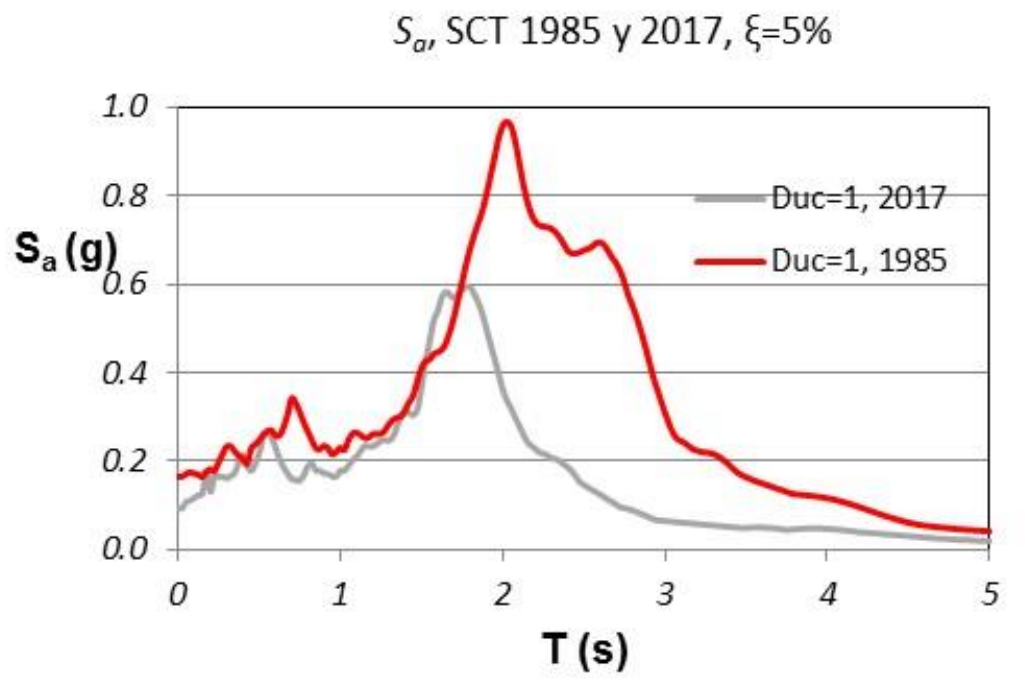

(a) Espectros elásticos $S_{a}$ para los registros SCT de 1985 y 2017

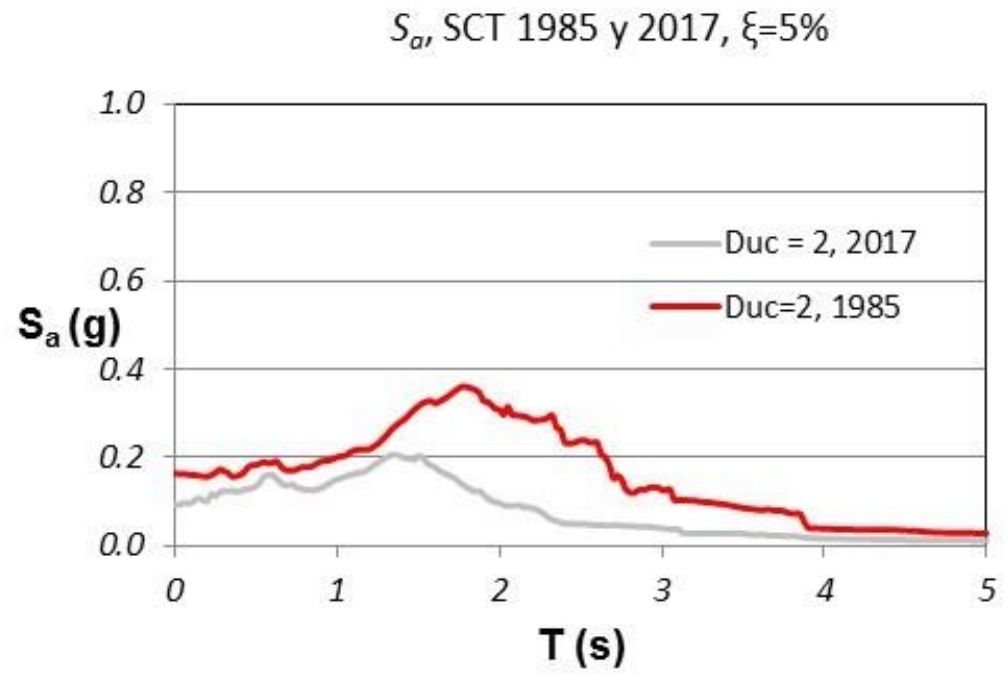

(b) Espectros inelásticos $S_{d}$ para $\mu=2$, para los registros SCT de 1985 y 2017

Figura 6. Ordenadas $S_{a}$ elásticas e inelásticas $(\mu=2)$, obtenidas con los registros de la estación SCT en los terremotos de 1985 y 2017 
La Fig. 7 muestra resultados de demandas espectrales de desplazamientos $S_{d}$ para los registros de la estación SCT de 1985 y 2017, tanto para la respuesta lineal, como para la correspondiente a la respuesta inelástica, $\mu=2$. La comparativa de demandas espectrales en ambos terremotos es semejante al caso mostrado en la Fig. 6 para $S_{a}$.

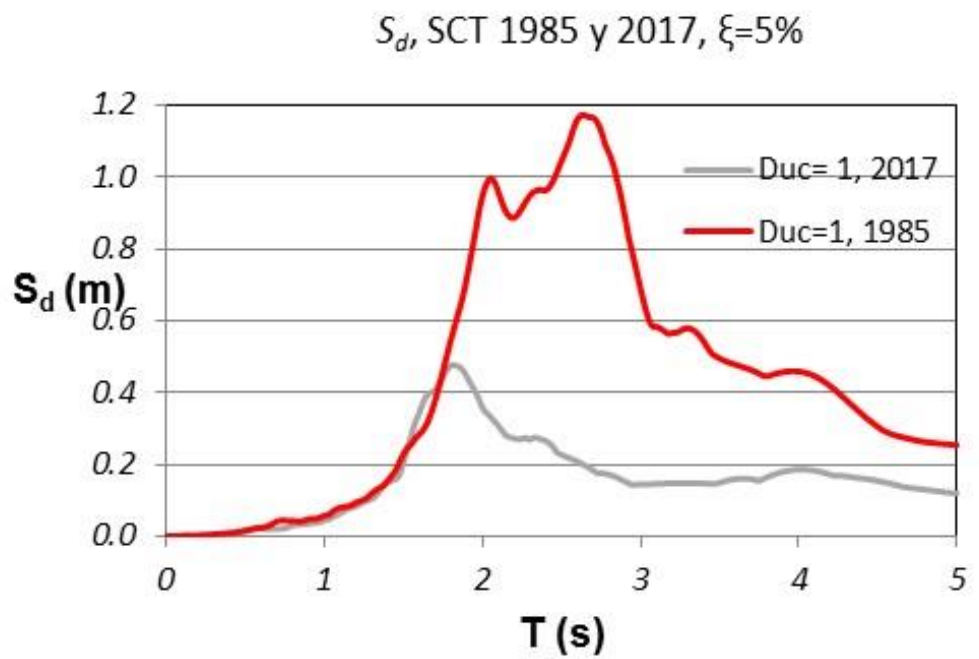

(a) Espectros elásticos $S_{d}$ para los registros SCT en 1985 y 2017.

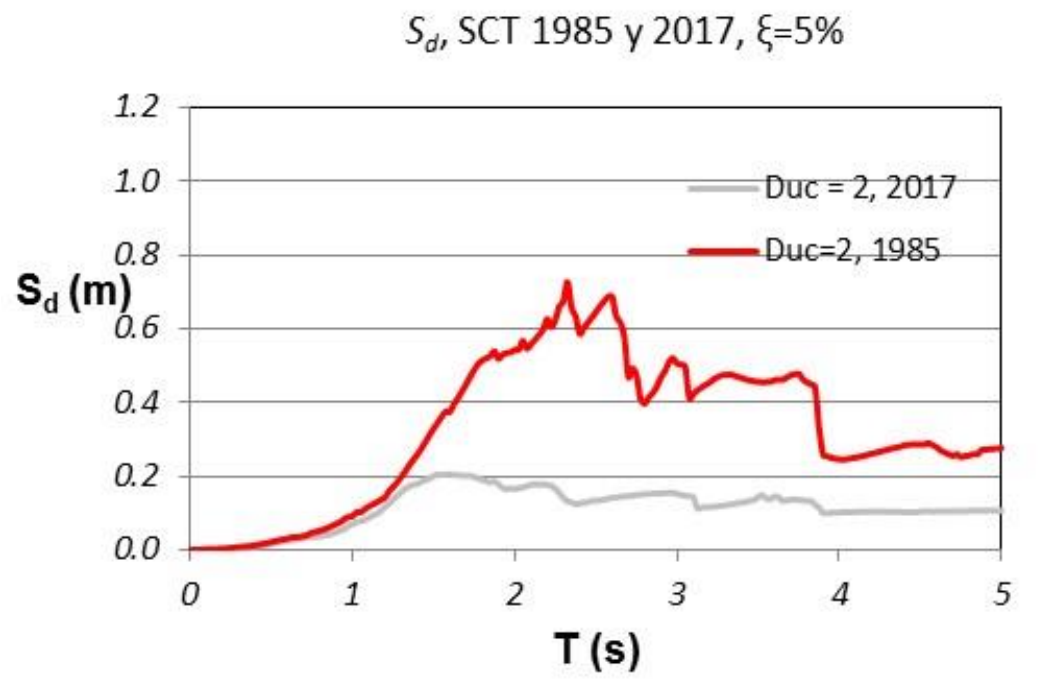

(b) Espectros inelásticos $S_{d}$ para $\mu=2$, para los registros SCT en 1985 y 2017

Figura 7. Ordenadas espectrales $S_{d}$ de respuesta lineal e inelástica $(\mu=2)$, obtenidas con los registros de la estación SCT en los terremotos de 1985 y 2017

La estación SCT, cuyos registros se emplean en este trabajo, no se ubica exactamente en la zona de derrumbes de edificios observados en el terremoto de 2017; sin embargo, no se encuentra lejos de la ubicación de algunos casos de derrumbes o daños severos observados en edificaciones en este terremotos, 
y se tiene la ventaja que se han obtenido registros de aceleraciones en esta estación tanto en el terremoto de 1985, como el de 2017. El empleo de estos registros permite intentar interpretar el comportamiento observado en edificaciones en ambos terremotos, en la zona de periodos fundamentales de edificaciones de interés.

Los resultados de respuestas espectrales mostrados en este trabajo y los daños y colapsos observados en la Ciudad de México en el terremoto del 19 de septiembre de 2017 llevan a preguntas cuyas respuestas son relevantes no solo para la interpretación de los daños y colapsos observados en este terremoto, sino también para conocer si procedimientos de diseño sísmico convencionales, como los especificados en las NTCS 2004 y NTCS 2017, son adecuados, y además para definir las estrategias de rehabilitación sísmica en las edificaciones dañadas por el terremoto de 2017. Si para el análisis del comportamiento de edificaciones que experimentaron los terremotos de 1985 y 2017, consideramos aquellas edificaciones construidas en suelos con características comparables al de la estación SCT, este análisis lleva a las siguientes preguntas de interés:

1. Si consideramos que edificios con colapsos en el terremoto de 2017, en su mayor parte ya estaban en el terremoto de 1985, y que las ordenadas espectrales para el registro de la estación SCT de 1985 son comparables a las del registro en esta estación en 2017 en la zona de periodos críticos, correspondientes a edificaciones en el intervalo de niveles menores a 10, ¿por qué ocurrió el colapso de edificios en el terremoto de 2017 y éstos no lo tuvieron en el terremoto de 1985 ?

2. Si las demandas espectrales $S_{a}$ y $S_{d}$ para los registros en la estación SCT, en el terremoto de 2017 fueron menores que las especificadas por la normativa por sismo vigente en la ciudad de México, y aun así se observaron colapsos y daños severos en edificaciones en este terremoto en zonas no muy lejanas a la estación SCT, ¿son adecuadas estas normativas para el diseño sísmico de nuevos edificios en la ciudad de México y/o para la rehabilitación por sismo de edificios existentes?

3. De la observación de daños de edificaciones en el terremoto de 2017 se concluye que la mayoría de las edificaciones que tuvieron derrumbes o daños en el terremoto de 2017 ya habían experimentado el terremoto de 1985. Por el contrario, en la mayor parte de edificaciones construidas después de 1985 no se observaron colapsos. ¿Cómo se explica este mejor comportamiento observado en edificaciones construidas después de 1985, respecto al comportamiento de las que experimentaron los terremotos de 1985 y $2017 ?$

Estas preguntas se tratan de responder en lo que sigue.

\section{INTERPRETACIÓN DE LOS DAÑOS Y COLAPSOS EN EDIFICACIONES OBSERVADOS EN LA CIUDAD DE MÉXICO EN EL TERREMOTO DEL 19 DE SEPTIEMBRE 2017}

Como se ha mostrado en este trabajo, la comparativa de respuestas espectrales de pseudo-aceleraciones y desplazamientos calculados para el registro en la estación SCT en este terremoto, y las demandas sísmicas de diseño especificadas por la NTCS (2004), no permite explicar las razones de los daños y colapsos observados en el terremoto de 2017. La evidencia en el terremoto de 2017 de que las edificaciones que sufrieron colapsos o daños graves en este terremoto, no habían tenido daños considerables en el terremoto del 19 de septiembre de 1985, sugiere la necesidad de explorar efectos en estructuras causados por terremotos que no se consideran en procedimientos de diseño sísmico convencionales en diversas prácticas de diseño sísmico en el mundo, incluyendo la de la Ciudad de México. Entre estos efectos está el efecto del 
daño acumulado por sismo en estructuras, cuando éstas se ven sometidas a más de un terremoto, como fue el caso de un número considerable de edificaciones en la Ciudad de México que tuvieron colapsos o daños severos en el terremoto del 19 de septiembre de 2017 y en las cuales no se observaron daños considerables en el terremoto del 19 de septiembre de 1985. Para este fin, en este trabajo además del análisis de respuestas espectrales, se emplea un índice de daño en estructuras debido a terremotos propuesto por el autor y que se describe brevemente en lo que sigue. Una descripción de este índice, así como de resultados de su aplicación para el análisis del comportamiento de edificaciones en diversos terremotos en el mundo se encuentra en Rodríguez (2015).

\section{Índice de daño por sismo}

Rodríguez (2015) ha propuesto el índice de daño, $I_{d}$, donde $I_{d}=1$ en el colapso y $I_{d}=0$ para el caso sin daños. Este índice se define como:

$$
I_{d}=\frac{\Gamma^{2} E_{H}}{\left(2 \pi \lambda h D_{r c}\right)^{2}}
$$

En la ec. (1), $E_{H}$ es la energía histerética, definida como el trabajo inelástico de un oscilador de un grado de libertad, cuando se analiza con un registro de aceleraciones. El cómputo de $E_{H}$ en este estudio emplea la regla de histéresis de Takeda modificada (Carr, 2011). El parámetro $\Gamma$ es el factor de participación. El parámetro $h$ es la altura de entrepiso de un edificio medida en metros, y el factor $\lambda$ es el empleado para estimar el periodo fundamental, $T$, con la siguiente expresión:

$$
T=\frac{n}{\lambda}
$$

donde $n$ es el número de niveles de un edificio.

El parámetro $D_{r c}$ es un valor seleccionado para una distorsión global de referencia en un edificio y se considera igual a 0.025 (Rodríguez, 2015). Este valor de $D_{r c}$ aplica a edificios diseñados con reglamentos modernos sismorresistentes. Esto lleva a que para casos de diseño en México con el factor de reducción $Q=2$, tanto en edificaciones existentes, como en nuevas en la Ciudad de México, el referido valor de $D_{r c}$ estaría sobrestimado, lo que llevaría a subestimar los valores calculados de $I_{d}$ (Rodríguez, 2016). En este caso los valores calculados de $I_{d}$ serían una cota inferior a los resultados que se obtendrían con valores menores que 0.025. El factor $Q$ es el valor del factor de reducción de fuerzas sísmicas para diseño especificado por la NTCS (2004). Una situación semejante de subestimar los valores calculados de $I_{d}$ ocurriría para los casos de edificaciones con irregularidades en planta o elevación, como por ejemplo el caso de "planta baja débil", que era característica de un número importante de daños severos o colapsos de edificaciones en el terremoto de 2017. Esta característica de subestimar los valores calculados de $I_{d}$ se debe a que en estas edificaciones irregulares las demandas de desplazamientos laterales en la estructura se concentran principalmente en la planta baja, causando distorsiones excesivas en este nivel (Pujol y Rodríguez, 2018).

En este trabajo se obtienen valores del índice $I_{d}$ para los registros de aceleraciones en la estación SCT de 1985 y 2017 anteriormente comentados. Para la fracción de amortiguamiento crítico, $\xi$, se emplea el valor 2\%, con base en las recomendaciones de Martinelli y Filippou (2009). Se considera una altura de entrepiso, $h$, típica de $3 \mathrm{~m}$. Para esta evaluación se consideran dos tipos de sistemas estructurales sismorresistentes, 
el sistema a base de marcos y el sistema dual (combinación de marcos y muros estructurales de concreto reforzado). Para el primer tipo de sistema se considera $\lambda=7 \mathrm{~s}^{-1}$, con el cual en algunos casos de estructuras muy flexibles (por ejemplo de losas planas sin vigas) podría estar sobrestimado, lo que llevaría a sobrestimar la rigidez lateral de estos edificios flexible y a subestimar los valores de $I_{d}$. Para los sistemas del tipo dual, los cuales se empezaron a emplear en México de manera más frecuente después de 1985, se considera de manera conservadora $\lambda=10 \mathrm{~s}^{-1}$. Estos valores de $\lambda$ son congruentes con valores típicos en México cuando se emplea la ec. (1) para el cómputo aproximado del periodo fundamental de un edificio de concreto reforzado. La Fig. 8 muestra en una gráfica los valores de $I_{d}$ calculados con la ec. (1) para edificios a base de marcos para el caso $\mu=2$, para el registro en la dirección EO obtenida en la estación SCT en 1985, estos resultados se muestran con línea gris. Se debe notar que la componente EO para esta estación fue más intensa que la de la componente NS. También esta figura muestra resultados del cómputo de $I_{d}$ para el registro obtenido en el terremoto de 2017 en la estación SCT para la dirección EO, los que se muestran con línea negra.

En la Fig. 8 se observa que los valores calculados para $I_{d}$ para el caso del terremoto de 1985 en estructuras existentes en esa época son congruentes con los daños y colapsos observados en ese evento, los cuales se concentraron en la zona de periodos de alrededor de $2 \mathrm{~s}$. Sin embargo, de acuerdo con los resultados de la Fig 8, los edificios a base de marcos analizados con el registro de la SCT en 2017, ver línea negra en la figura, no habrían tenido daños graves o colapsos en el terremoto de 2017, lo que no es congruente con lo observado en edificios en este terremoto, por lo que estos resultados se vuelven a evaluar más adelante empleando otras hipótesis.

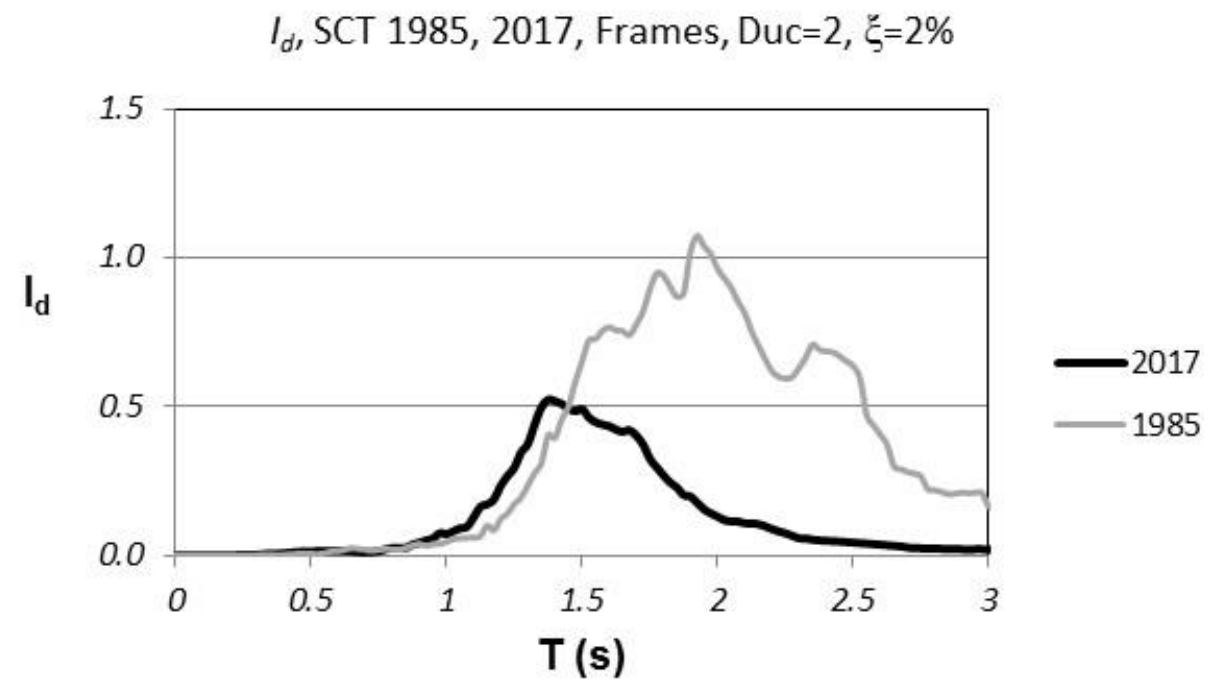

Figura 8. Índice de daño en edificaciones a base de marcos calculados para los registros de la estación SCT en los terremotos de 1985 y 2017

La Fig. 9 muestra resultados del cómputo de índice $I_{d}$ para el caso de edificios duales considerando el registro de la SCT de 1985, mostrados con línea gris, así como para el registro de 2017, mostrado con línea negra. De acuerdo con los resultados de la Fig. 9, edificios con sistema estructural del tipo dual, construidos antes de 1985 no habrían tenido colapsos o daños severos en el terremoto de ese año, ver línea gris en la Fig. 9. Tampoco edificios con sistema dual construidos después de 1985 que experimentaron el terremoto de 2017 habrían tenido colapsos o daños severos, ver línea negra en la Fig. 9. Este último caso es congruente con lo observado en el terremoto de 2017 para el caso de edificaciones con sistema dual posteriores a 1985, pero queda por explicar el comportamiento de edificaciones con sistema dual anteriores a 1985 que 
experimentaron los terremotos de 1985 y 2017, las que de acuerdo con los resultados de la Fig. 9 no habrían tenido colapsos o daños severos. Como en el caso de edificios a base de marcos existentes antes de 1985, que experimentaron ambos terremotos, de 1985 y 2017, el caso de sistemas duales que experimentaron ambos terremotos se debe revisar, lo que se lleva a cabo en lo que sigue.

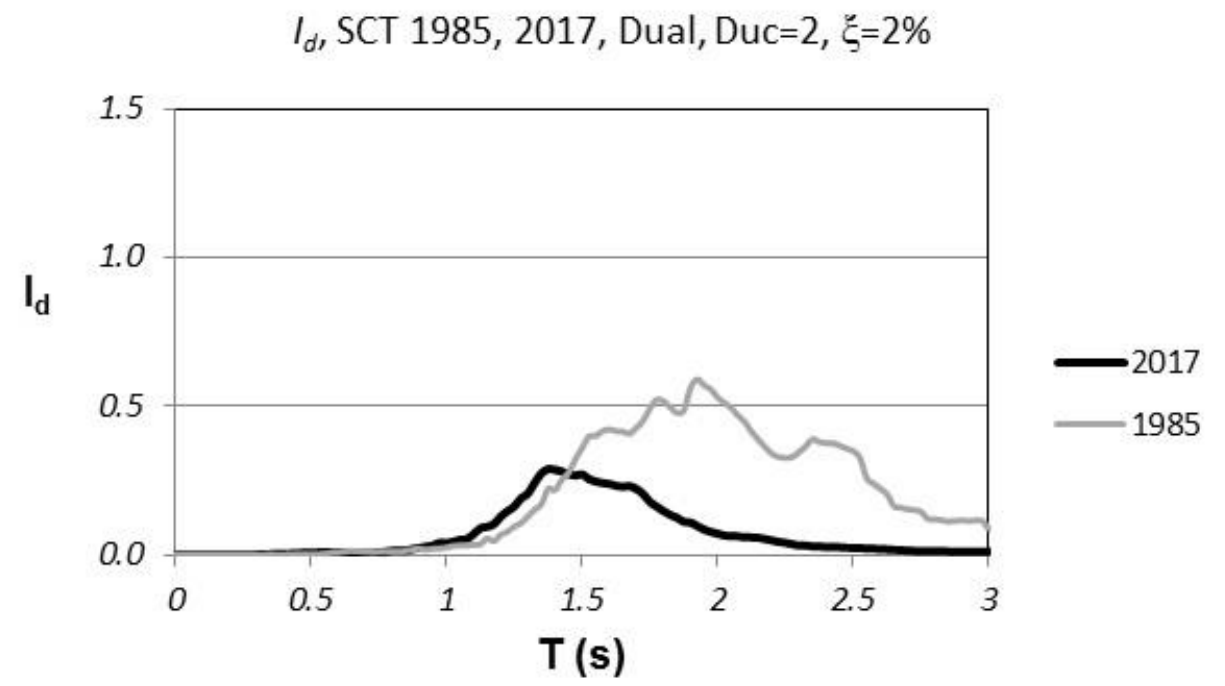

Figura 9. Índice de daño en edificaciones a base de sistema dual calculados para los registros de la estación SCT en los terremotos de 1985 y 2017

\section{Respuesta de edificaciones considerando la combinación de movimientos en los terremotos de 1985 y 2017}

La Fig. 10 muestra el registro de aceleraciones que se obtendría combinando los registros en SCT obtenidos en SCT en 1985 y en 2017. La parte de la izquierda de la Fig. 10 corresponde al registro de 1985 y la parte derecha al registro de 2017.

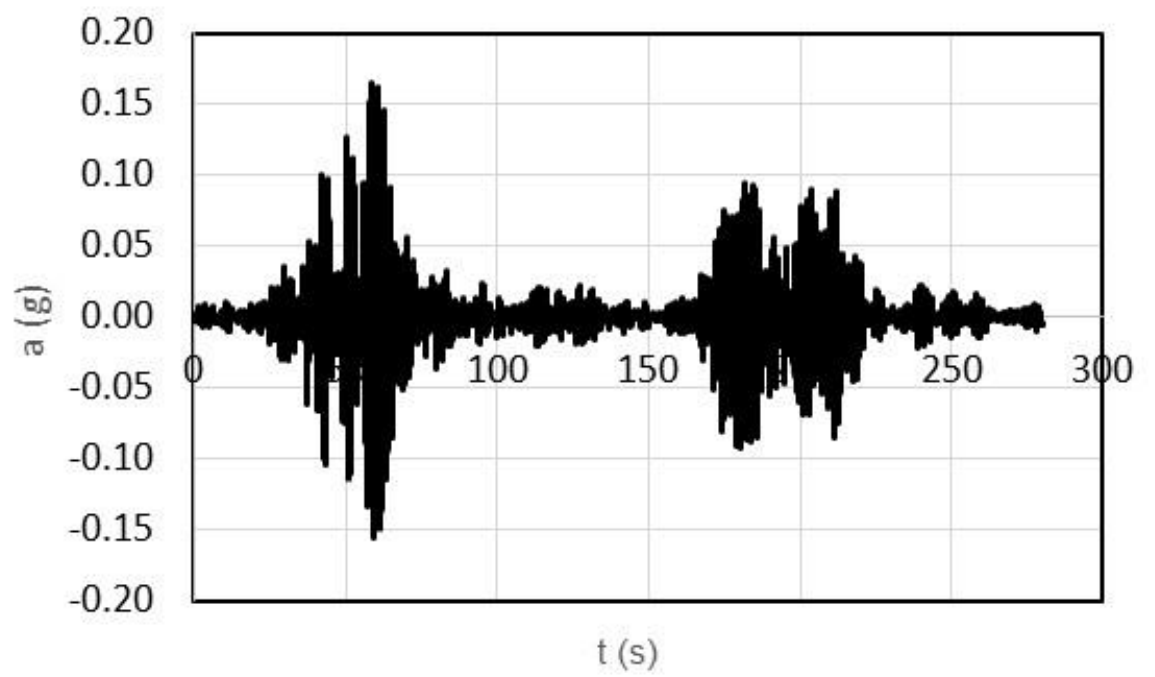

Figura 10. Registro resultante de combinar los registros de aceleraciones de la estación SCT componente EO obtenidos en los terremotos de 1985 y 2017 
La Fig. 11 muestra resultados que se identifican con la línea gris, obtenidos para el cómputo del índice $I_{d}$ correspondientes al caso de marcos cuando se emplea el registro de aceleraciones combinado de 1985 y 2017, mostrado en la Fig. 10. Estos resultados corresponden al caso $Q=2$, este valor de 2 es el que mayormente se emplea para el diseño sísmico de edificaciones de concreto reforzado en la Ciudad de México. También la Fig. 11 muestra valores calculados para este índice cuando los edificios a base de marcos analizados están sometidos sólo al registro de la SCT en 2017, línea negra en la Fig. 11. Estos últimos valores también se han mostrado en la Fig. 8.

De la Fig. 11 se concluye que edificaciones a base de marcos que fueron construidas después de 1985, que experimentaron sólo el terremoto de 2017, no muestran valores del índice $I_{d}$ correspondientes a colapsos o daños severos, lo que no es congruente con lo observado en este terremoto. Sin embargo, si se considera las edificaciones que experimentaron ambos terremotos, de 1985 y 2017, éstas habrían colapsado o tenido daño severos para los casos en la zona de periodos críticos mostrado, como lo muestran los resultados con línea gris en la Fig. 11. Estos resultados son congruentes con los daños y colapsos observados en edificaciones que experimentaron en la ciudad de México los terremotos de 1985 y 2017. Es de interés que de acuerdo con la Fig. 11, cuando se considera el efecto combinado de ambos terremotos, para pequeños incrementos de periodos fundamentales en el intervalo de $1 \mathrm{~s}$ a $1.5 \mathrm{~s}$, el índice de daño aumenta de manera considerable, lo que es congruente con los mayores daños observados en el terremoto de 2017 en edificaciones a base de marcos de mediana altura, en dicho intervalo de periodos, al que le correspondería los casos de edificaciones en el intervalo alrededor de 6 y10 niveles. Se debe observar que este intervalo de periodos, entre $1 \mathrm{~s}$ y $1.5 \mathrm{~s}$, corresponde al intervalo de periodos dominantes del suelo donde se ubicaron la mayor parte de colapsos, ver Fig. 1.

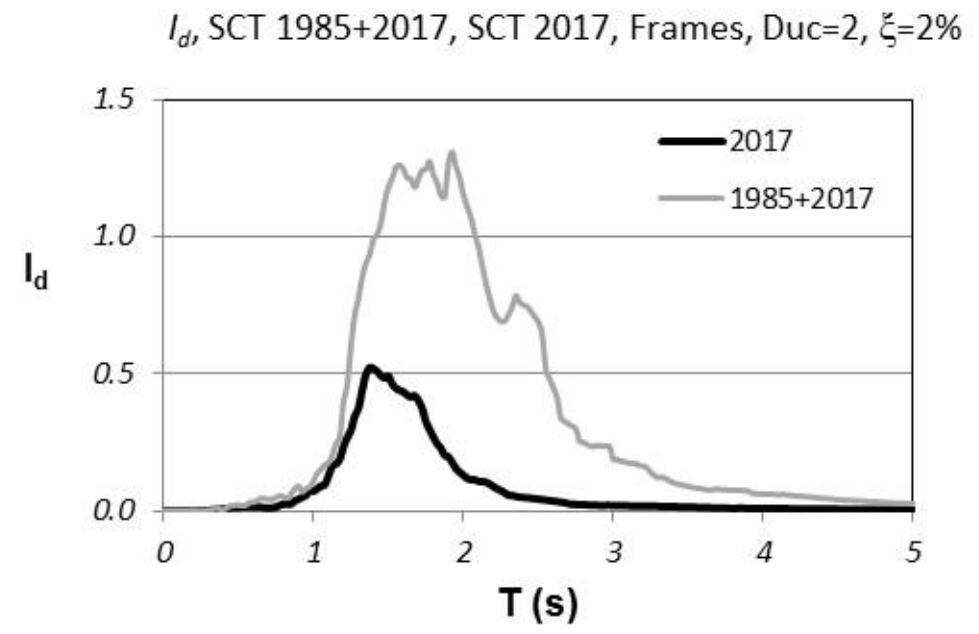

Figura 11. Índice de daño en marcos, calculado para el efecto combinado de los registros obtenidos en la estación SCT en los terremotos de 1985 y 2017, y para sólo el registro de esta estación en 2017

La Fig. 12 muestra resultados del mismo tipo de los de la Fig. 11, en este caso se evalúa el índice $I_{d}$ para el caso de edificaciones con sistema estructural del tipo dual. Los resultados muestran que edificaciones con sistema sismorresistente del tipo dual, existentes antes de 1985, no habrían colapsado o tenido daños severos en 1985, ver línea gris en la Fig. 9, y tampoco en 2017 con el tipo de efecto combinado de los registros de 1985 y 2017, ver línea gris en la Fig. 12. Tampoco los resultados indican potencial de colapso o daños 
severos en edificaciones con sistema dual construidas después de 1985, que experimentaron sólo el terremoto de 2017 ver línea negra en la Fig. 12. Estos resultados son congruentes con el comportamiento prácticamente sin daños observado en el terremoto de 2017 en edificaciones de concreto reforzado con sistema dual; sin embargo, se debe tomar en cuenta que el sistema dual se emplea en México principalmente después de 1985, es decir este sistema estructural en general no ha estado expuesto al tipo de efecto combinado de los registros de 1985 y 2017. Este buen comportamiento de edificios con sistema dual también es congruente con el comportamiento de edificaciones con muros estructurales de concreto reforzado observado en diversos terremotos en el mundo (Rodríguez, 2015). Aun cuando la densidad de muros estructurales en los sistemas duales que se emplean en México es bastante menor que las densidades típicas por ejemplo en edificaciones en Chile, los resultados de la Fig 12 y el comportamiento observado en este tipo de edificios en el terremoto de 2017, muestran las ventajas del empleo de muros estructurales de concreto reforzado en edificios, para evitar el colapso de éstos en terremotos, lo que también ha sido observado por Rodríguez (2016) para el caso de edificaciones en la Ciudad de México.

$I_{d}$, SCT 1985+2017, SCT 2017, Dual, Duc $=2, \xi=2 \%$

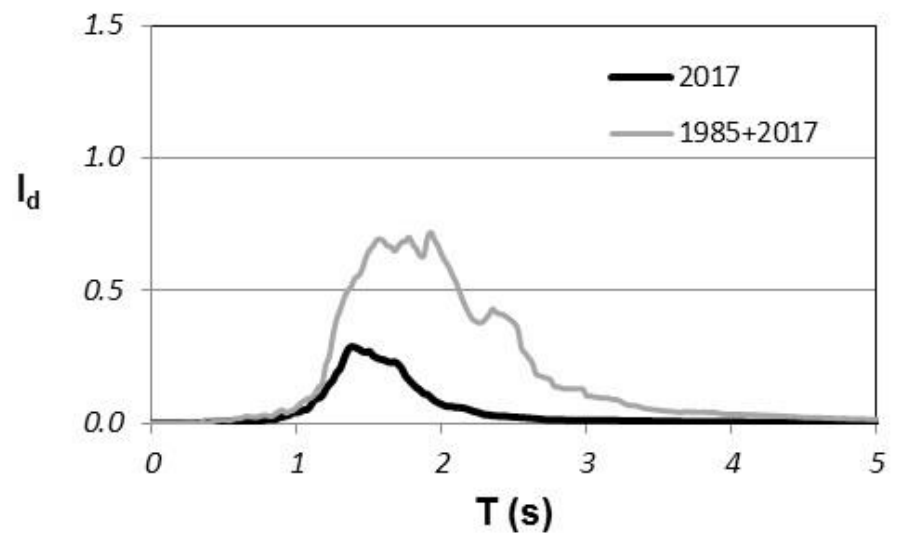

Figura 12. Índice de daño para el sistema dual, calculado para el efecto combinado de los registros de la estación SCT en los terremotos de 1985 y 2017, y para sólo el registro de esta estación en 2017

\section{CONCLUSIONES}

En este trabajo se intenta responder a las tres preguntas que se han planteado en esta investigación.

1. ¿Por qué en el terremoto de 2017 ocurrieron colapsos o daños severos en edificaciones que ya habían experimentado sin colapsos el terremoto de 1985 ?

Los resultados mostrados en este trabajo indican que para una mejor interpretación de la vulnerabilidad de estructuras se debe considerar el efecto de daño acumulado cuando las estructuras experimentan más de un terremoto fuerte, como fue el caso de las edificaciones con colapsos o daños severos observados en la Ciudad de México en el terremoto de 2017. El índice de daño $I_{d}$, empleado en este estudio, toma en cuenta el daño acumulado, y sus resultados son congruentes con los daños y colapsos observados en edificaciones que experimentaron en la Ciudad de México los terremotos de 1985 y 2017. Se debe tener en cuenta que el problema de la inseguridad de estas edificaciones se agrava con la insuficiente rigidez lateral que caracteriza 
a los sistemas estructurales a base de marcos, también por la existencia de irregularidades en ellas, como fueron los numerosos casos observados de edificaciones a base de marcos con "planta baja débil", así como con el detallado de refuerzo en estas estructuras, el cual está por debajo de estándares internacionales de diseño en zonas sísmicas.

2. ¿Son adecuadas las normativas para el diseño sísmico de nuevos edificios en la Ciudad de México y/o para la rehabilitación por sismo de edificios existentes?

Si consideramos que el terremoto del 19 de Septiembre de 2017 tuvo una intensidad por debajo del sismo de diseño, los resultados encontrados en este trabajo indica la necesidad de cambios en la práctica de diseño por sismo en México para lograr un comportamiento aceptable de edificaciones en terremotos que ocurran en el futuro, así como para definir estrategias de rehabilitación sísmica de edificaciones. Como se ha mostrado en este trabajo, es deseable que normativas de diseño por sismo consideren el efecto de daño acumulado en edificaciones debido a terremotos; sin embargo, esto implica posibles modificaciones importantes en la práctica convencional de diseño sísmico que podrían requerir un tiempo considerable, por lo que al menos se debe considerar los siguientes cambios en el diseño sísmico en México, tanto para edificaciones nuevas, como para la rehabilitación de edificaciones dañadas en el terremoto de 2017.

Las edificaciones de concreto reforzado en zonas de mayor sismicidad tanto en la Ciudad de México, como en otras zonas sísmicas del país, se deberían diseñar con los requisitos de diseño por sismo correspondientes a elementos estructurales especiales especificados en el ACI 318. Esto implica que normativas de construcción, en la Ciudad de México y en el país, no deberían permitir en zonas sísmicas el empleo de estructuras con ductilidad limitada, como es el caso de estructuras diseñadas con $Q=2$, valor que típicamente se considera para el diseño sismorresistente de la mayoría de edificaciones en México. Además, la normativa de diseño por sismo en la Ciudad de México debería limitar el empleo de edificaciones a base de marcos de concreto reforzado, y también esta normativa debería reconocer las ventajas del empleo de edificaciones con muros estructurales de concreto reforzado, como ocurre por ejemplo en la práctica de países con alta actividad sísmica, como son los casos de Japón y Chile.

Se debe tener en cuenta que la filosofía de diseño por sismo de algunas normativas en el mundo, como es el caso de México, se basa en aceptar daños estructurales para el sismo de diseño. Estructuras esenciales, por ejemplo, hospitales, no se deberían diseñar con esta filosofía, en estos casos se deben emplear soluciones estructurales innovadoras que permitan que este tipo de estructuras sigan operando aun después de un terremoto. Soluciones de este tipo son el empleo de aisladores de base, como es la práctica en algunos países de Latinoamérica como Chile y Perú, o el empleo de elementos disipadores de energía.

3. ¿Cómo se explica el mejor comportamiento observado en edificaciones construidas después de 1985, respecto al comportamiento de las que experimentaron los terremotos de 1985 y 2017 ?

Para responder esta pregunta se considera dos factores. El primero se refiere al efecto de la duración de un terremoto, que causa el daño acumulado que se ha comentado en este trabajo. El segundo factor es el efecto del empleo de muros estructurales en edificios. Referente al primer factor, se ha mostrado en este trabajo que el daño acumulado en edificaciones a base de marcos es un factor importante para explicar los colapsos y daños severos observados en estas edificaciones en el terremoto de 2017. Este efecto no existió en el caso de edificaciones a base de marcos construidas después de 1985, lo que ayuda a explicar el comportamiento observado sin daños importantes en estas edificaciones en el terremoto de 2017, lo que es congruente con 
los valores del índice de daño $I_{d}$ que se muestran en este trabajo para el caso de edificios a base de marcos que experimentaron sólo el terremoto de 2017.

Respecto al segundo factor referente al empleo de edificios en México con sistemas duales, los resultados del cómputo del índice de daño $I_{d}$ mostrados en este trabajo, indican que aun considerando el efecto combinado de los terremotos de 1985 y 2017, las edificaciones con sistema dual no tienen potencial de colapso, lo que también es congruente con la ausencia de colapsos en edificaciones con sistema dual observados en el terremoto de 2017. Estos resultados muestran las ventajas de los sistemas rígidos respecto a los flexibles, lo que se refleja en las ventajas de sistemas estructurales del tipo dual respecto a los sistemas a base de marcos. También debe mencionarse que después de 1985, la normativa por sismo en la Ciudad de México cambió respecto a la existente en esa época, requiriendo más resistencia y rigidez lateral en las edificaciones, lo cual es un factor adicional para interpretar el mejor comportamiento de estas edificaciones en el terremoto de 2017 respecto al observado en edificaciones en el terremoto de 1985.

\section{AGRADECIMIENTOS}

El autor agradece al grupo de sus estudiantes de Posgrado de Ingeniería de la UNAM, que no solo lo acompañaron por varios días en la evaluación de los daños en la Ciudad de México causados en edificaciones por el terremoto del 19 de septiembre de 2017, sino también contribuyeron a obtener información para llevar a cabo este estudio. Estos estudiantes fueron Dandy Roca, Luis Aguilar, Giovani Quintino e Isabel Piedrahita. Se agradece al Profesor José I. Restrepo de la Universidad de California en San Diego y al Profesor Santiago Pujol de Purdue University, por sus comentarios para mejorar el borrador de este trabajo. También se agradece al M en Sc Francisco García Álvarez y al Dr. Luciano Fernández, ambos de la Sociedad Mexicana de Ingeniería Estructural (SMIE), por compartir información sobre los daños en edificaciones debidos a este terremoto. Los registros de 2017 empleados en este trabajo fueron proporcionados por la Unidad de Instrumentación Sísmica del Instituto de Ingeniería de la UNAM.

\section{REFERENCIAS}

Berrón, R. (2018). "Sismo del 19 Septiembre de 2017" (The September 19, 2017 Earthquake). XXI Congreso Nacional de Ingeniería Estructural, Campeche, México.

Carr, A. (2011), "RUAUMOKO, Computer Program Library", University of Canterbury, Department of Civil Engineering.

Martínez-Gonzalez J., Lermo J., Vergara F., y Ramos E., (2015). “Avances en la Zonificación Sísmica de la Ciudad de México y zona de Chalco, Edo de Méx., propuesta de nuevo mapa de periodos dominantes para las NTC para Diseño por Sismo del Reglamento del DF". Memorias del XX Congreso Nacional de Ingeniería Sísmica, Acapulco, Guerrero,

Norma Técnica Complementaria por Sismo (2004). Gaceta oficial del Distrito Federal.

Norma Técnica Complementaria por Sismo (2017). Gaceta oficial de la Ciudad de México.

Martinelli, P., y Filippou, F. (2009), "Simulation of the shaking table test of a seven-story shear wall building”, Earthquake Engineering-Structural Dynamics, 38, 587-607. DOI: 10.1002/eqe.897 
Martínez-Gonzales J., Lermo J., Vergara-Huerta F., y Ramos-Pérez E. (2015), “Avances en la zonificación sísmica de la Ciudad de México y zona de Chalco, Edo. de México, propuesta de nuevo mapa de periodos dominantes para las NTC para Diseño por sismo del Reglamento del DF". Memorias XX Congreso Nacional de Ingeniería Sísmica, Acapulco, Guerrero.

Pujol S., y Rodríguez M. (2018), "Desempeño de elementos no estructurales en edificios en el terremoto del 19 de septiembre en 2017 en la Ciudad de México", enviado para publicación en el número especial de la Revista Ingeniería Sísmica.

Rodríguez, M, Alarcón P, y Machicao R (1999). "Evaluación del comportamiento sísmico de edificaciones de mampostería en los sismos de Michoacan de 1985 y 1997”, Revista Ingeniería Sísmica, No. 59.

Rodríguez M. E. (2015), “Evaluation of a proposed damage index for a set of earthquakes”, Earthquake Engineering \& Structural Dynamics, 44, 1255-1270. DOI: 10.1002/eqe.2512

Rodríguez, M. E. (2016) "Una revisión crítica de la práctica de diseño por sismo de estructuras en Mexico", Revista Ingeniería Sísmica, No 94, 27-48. 